\title{
Methanol on Enceladus
}

\author{
Robert Hodyss, ${ }^{1}$ Christopher D. Parkinson, ${ }^{2}$ Paul V. Johnson, ${ }^{1}$ Julie V. Stern, ${ }^{1}$ \\ Jay D. Goguen, ${ }^{1}$ Yuk L. Yung, ${ }^{3}$ and Isik Kanik ${ }^{1}$ \\ Received 26 May 2009; revised 5 August 2009; accepted 11 August 2009; published 15 September 2009.
}

[1] Near infrared spectra of the surface of Enceladus returned by Cassini show the presence of an absorption feature at $3.53 \mu \mathrm{m}$, ascribed by Brown et al. (2006) to "short chain organics," and by Newman et al. (2007) to hydrogen peroxide. We assign this feature tentatively to methanol. Variations in the peak position of the feature suggest that methanol in the "tiger stripes" region may be segregated from the water ice, and not homogeneously distributed in the ice matrix. The photolytic destruction of methanol implies that methane or methanol itself must be continually deposited on the surface. On Enceladus, methanol may be generated photochemically from a mixed methane/water ice, or deposited from the plume itself. The variation in the concentration of methanol over the surface could be used to distinguish between these two processes. Citation: Hodyss, R., C. D. Parkinson, P. V. Johnson, J. V. Stern, J. D. Goguen, Y. L. Yung, and I. Kanik (2009), Methanol on Enceladus, Geophys. Res. Lett., 36, L17103, doi:10.1029/2009GL039336.

[2] Recent near infrared spectra of the surface of Enceladus obtained by Cassini show the presence of an absorption feature in the $\mathrm{C}-\mathrm{H}$ stretch region at $3.53 \mu \mathrm{m}$ [Brown et al., 2006]. Brown et al. [2006] ascribe this feature to "short chain organics." Newman et al. [2007] attribute this feature to hydrogen peroxide, although the observed width of the feature is significantly narrower than is seen in spectra of hydrogen peroxide. A careful analysis of hydrogen peroxide peak widths and positions under a variety of conditions has led Loeffler and Baragiola [2009] to conclude that hydrogen peroxide is not the carrier of the $3.53 \mu \mathrm{m}$ band. Here, we present evidence that methanol is the origin of the $3.53 \mu \mathrm{m}$ band. Methanol may be delivered to the surface by deposition of methanol present in the plume, or it may be formed photochemically from mixed ices of water and methane. The $3.53 \mu \mathrm{m}$ feature varies in both peak position and intensity over the surface of Enceladus, and these variations might be used to determine the source of the methanol.

[3] Figure 1 compares reflectance spectra acquired by Cassini VIMS of Enceladus with transmission spectra of ices obtained in our laboratory. The spectrum of a $1 \%$ mixture of methanol in crystalline water ice at $140 \mathrm{~K}$ is an excellent match to the feature at $3.53 \mu \mathrm{m}$ in the global

\footnotetext{
${ }^{1}$ Jet Propulsion Laboratory, California Institute of Technology, Pasadena, California, USA.

${ }^{2}$ Department of Atmospheric, Oceanic, and Space Sciences, University of Michigan, Ann Arbor, Michigan, USA.

${ }^{3}$ Division of Geological and Planetary Sciences, California Institute of Technology, Pasadena, California, USA.
}

Copyright 2009 by the American Geophysical Union. 0094-8276/09/2009GL039336\$05.00 average spectrum of Enceladus, both in peak position and line width. On Enceladus, this band varies in position from 3.53 to $3.56 \mu \mathrm{m}$, with FWHM between 0.02 to $0.03 \mu \mathrm{m}$ [Newman et al., 2007]. The FWHM of our methanol band is $0.02 \mu \mathrm{m}$, in contrast to the FWHM of $\mathrm{H}_{2} \mathrm{O}_{2}$ in water ice, which varies from 0.03 to $0.15 \mu \mathrm{m}$ [Newman et al., 2007], suggesting the feature is more likely due to methanol. We saw little variation in the shape or position of this band with temperature and phase of the water ice matrix. In the spectra of the "tiger stripes" region on Enceladus, the peak position of the band has shifted to $3.55 \mu \mathrm{m}$. As shown in Figure 1, this shift is partly replicated by a thin film of pure, amorphous methanol ice. This may indicate that the methanol in this region is segregated from the water ice into domains of pure methanol, or that a different matrix is present in the warmer regions, perturbing the position of the band. In the spectrum of the region between the tiger stripes, the $3.55 \mu \mathrm{m}$ feature is broadened, possibly indicating the presence of both segregated methanol and methanol isolated in the water ice matrix. Ion irradiation may also be capable of altering the width and intensity of this band [Brunetto et al., 2005; Palumbo et al., 1999].

[4] Methanol exhibits two other bands in the near infrared, at 2.27 and $2.34 \mu \mathrm{m}$. Sandford and Allamandola [1993] measured the integrated absorbances of these features in a water-rich matrix. The $2.27 \mu \mathrm{m}$ band has a band strength of $4 \times 10^{-19} \mathrm{~cm} \cdot$ molecule $^{-1}$, and the $2.34 \mu \mathrm{m}$ band has a band strength of $6 \times 10^{-20} \mathrm{~cm} \cdot$ molecule ${ }^{-1}$. These bands are significantly weaker than the $3.53 \mu \mathrm{m}$ band $(7.5 \times$ $10^{-18} \mathrm{~cm} \cdot$ molecule ${ }^{-1}$; the "weak IS mix" from Hudgins et al. [1993]). Although the $2.27 \mu \mathrm{m}$ band might be detectable in the highest SNR ground based spectra [Emery et al., 2005], another feature they ascribe to $\mathrm{NH}_{3}$ covers the 2.235 to $2.275 \mu \mathrm{m}$ wavelength range. It is plausible that the $2.27 \mu \mathrm{m}$ methanol feature contributes part of this observed absorption. The weaker $2.34 \mu \mathrm{m}$ feature is not expected to be detectable and was not detected in the Emery spectra.

[5] On Enceladus, methanol may be present within the warmer interior, from either a primordial source or from in situ formation [Matson et al., 2007], and could then be released in the plume. In either case, methanol would condense on the surface, leading to the observed feature. This hypothesis is strengthened by recent data from the Cassini INMS indicating trace quantities of small organic compounds in the plume [Waite, 2008].

[6] Another possible source of methanol on Enceladus is photochemical generation from water and methane. Both water and methane are known components of the Enceladus plume [Waite et al., 2006], and could thus be deposited on the surface. Photolysis of methane/water ices has been shown to produce methanol, through the reaction of hydroxyl and methyl radicals formed through photolysis and hydrogen atom abstraction [Hodyss et al., 2009]. The warmest areas 


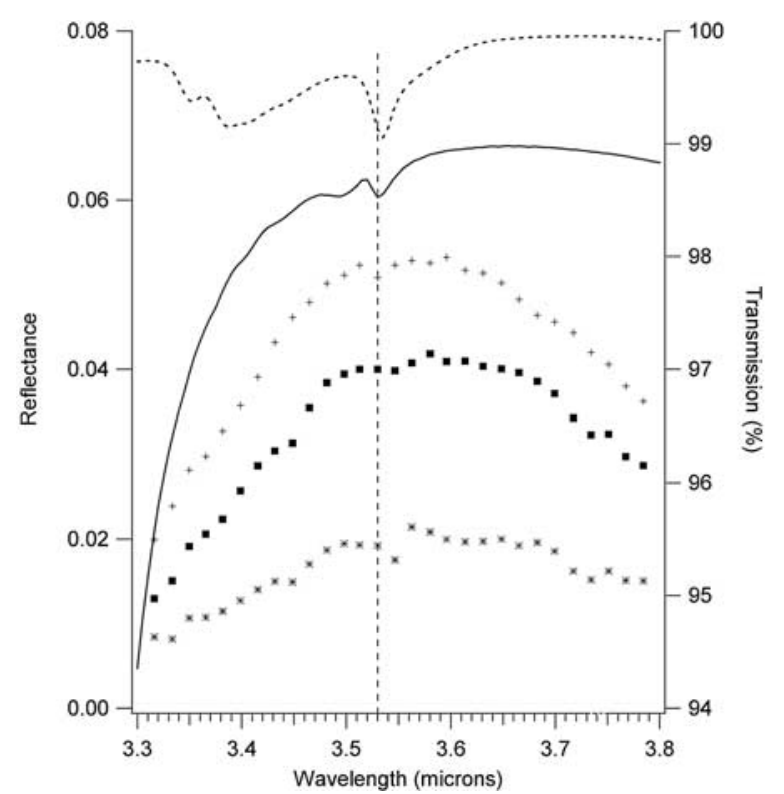

Figure 1. Laboratory transmission and Cassini VIMS reflectance spectra. The vertical dashed line is at $3.530 \mu \mathrm{m}$. Dashed line: pure methanol at $100 \mathrm{~K}$; solid line: $1 \%$ methanol in crystalline water at $140 \mathrm{~K}$; crosses: Enceladus global average (methanol band position $3.530 \mu \mathrm{m}$ ); squares: Enceladus around tiger stripes (methanol band position $3.547 \mu \mathrm{m}$ ); stars: Enceladus tiger stripes (methanol band position $3.547 \mu \mathrm{m})$. The $1 \%$ methanol in water sample was deposited at $20 \mathrm{~K}$ at a rate of $2.5 \mu \mathrm{m} /$ hour, and then warmed to $140 \mathrm{~K}$ at approximately $1 \mathrm{~K} / \mathrm{min}$. For a detailed description of the location on Enceladus of the tiger stripes spectra, see Brown et al. [2006, Figure 1A].

of the tiger stripes have a temperature inferred to be at least 145K [Spencer et al., 2006], but other regions of Enceladus should be at temperatures controlled by insolation, ranging from 33 to $75 \mathrm{~K}$ [Spencer et al., 2006]. These lower temperatures should allow for condensation of some fraction of the plume methane onto the surface. Experiments have shown detectable methane in co-condensed methane/ water mixtures up to $74 \mathrm{~K}$ [Bernstein et al., 2006]. Subsequent photochemistry could then lead to the formation of methanol, and the $3.53 \mu \mathrm{m}$ band.

[7] Methane is not expected to be spectrally detected in the surface ice at the $1.6 \pm 0.4 \%$ concentration measured by INMS in the plumes. Emery et al. [2005] use high SNR spectra with better spectral resolution than VIMS to put an upper limit of 2-7 wt\% methane in Enceladus surface ice based on their spectra at 2.32 and $2.38 \mu \mathrm{m}$ where interference from water ice absorption features is minimal. At $3.32 \mu \mathrm{m}$, the methane absorption on Enceladus is not detected because it is masked by the strong absorption from the shoulder of the $3 \mu \mathrm{m}$ water ice band. Although methane also has an absorption feature near $3.53 \mu \mathrm{m}$, it is an order of magnitude weaker than the methanol feature $\left(\mathrm{A}\left(\mathrm{CH}_{3} \mathrm{OH}\right)\right.$ $\sim 7.5 \times 10^{-18} \mathrm{~cm} \mathrm{~mol}^{-1}$ [Hudgins et al., 1993]; $\mathrm{A}\left(\mathrm{CH}_{4}\right)$ $\sim 6 \times 10^{-19} \mathrm{~cm} \mathrm{~mol}^{-1}$ [Grundy et al., 2002]). Thus the lack of detection of methane features anywhere in the spectrum is consistent with the fidelity of the observed spectra and the INMS methane concentration in the plume. Conversion to methanol allows the $3.53 \mu \mathrm{m}$ absorption to become detect- able due to the increased intrinsic strength (A value) of the methanol band.

[8] Since hydroxyl radicals are continually produced by photolysis of the water ice, all methanol should eventually be oxidized to carbon dioxide [Hodyss et al., 2009], if not shielded by continually deposited materials from the plume. The reverse reaction, hydrogenation of $\mathrm{CO}_{2}$ to formaldehyde, methanol, or methane does not occur in irradiated ices [Strazzulla et al., 2005]. Carbon dioxide is therefore a sink, and has in fact been detected on Enceladus [Brown et al., 2006]. Methanol must be continually deposited onto the surface by the plume, or be continually produced on the surface through photochemical oxidation of deposited methane for the $3.53 \mu \mathrm{m}$ band to be extant. Parkinson et al. [2007] estimate the flux of water accreting on the surface is at least $10^{-5} \mathrm{~cm} /$ year. Is this a sufficient rate to shield the methanol from complete oxidation? From the photodestruction cross-section given by Cottin et al. [2003] $\left(2.7 \times 10^{-19}\right.$ $\mathrm{cm}^{2}$ ) and the flux of far ultraviolet radiation at Enceladus $\left(1.1 \times 10^{12}\right.$ photons $\mathrm{cm}^{-2} \mathrm{~s}^{-1}$ at $4.4-12.4 \mathrm{eV}$ [Madey et al. , 2002, Table 1], we can calculate a half life for methanol of 27 days. Water ice is strongly absorbing in the ultraviolet at wavelengths shorter than approximately $200 \mathrm{~nm}$ where photons have sufficient energy to photolyze water. For instance, below $155 \mathrm{~nm}, 90 \%$ of the light is absorbed within the top $0.1 \mu \mathrm{m}$ of a water ice surface [Cottin et al., 2003], a thickness which is deposited in 37 days on Enceladus at the flux given by Parkinson et al. [2007]. From these considerations it seems clear that the deposited water ice should provide sufficient shielding to protect methanol from complete oxidative destruction.

[9] It is important to note that photolysis of the surface ices is not the only form of radiation processing that will occur on Enceladus. Chemistry may also be induced by charged particle irradiation, and sputtering by both charged particles and ultraviolet photons will play a role in altering the surface. In general, the chemical effects of charged particles will be similar to that of ultraviolet photons for the water ice-organic mixtures we describe here. Charged particles and ultraviolet photons will both produce hydroxyl radicals that will lead to oxidative chemistry and eventual conversion of organics to $\mathrm{CO}_{2}$. However, charged particle irradiation will differ from photolysis in its distribution across the surface, and in its penetration depth into the ice. Enceladus is irradiated by magnetospheric protons at a energy flux of approximately $1 \times 10^{12} \mathrm{eV} \mathrm{cm}^{-2} \mathrm{~s}^{-1}$, corresponding to $1 \times 10^{7}$ ions $\mathrm{cm}^{-2} \mathrm{~s}^{-1}$ at $100 \mathrm{keV}$ [Loeffler et al., 2006]. The energy flux of far ultraviolet radiation given above is approximately $9 \times 10^{12} \mathrm{eV} \mathrm{cm}^{-2} \mathrm{~s}^{-1}$ (calculated as the average photon energy times the flux), and so ultraviolet photolysis should be the more important process.

[10] We have described two possible sources of methanol on the surface of Enceladus, viz., 1) deposition by the plume from the interior, and 2) generation photochemically on the surface from water and methane. These two scenarios will lead to different distributions of methanol on the surface, shown schematically in Figure 2. The composition of the condensing gasses on the surface is related to the temperature of the surface, which is warmer closer to the vent. In scenario 1 , methanol will be deposited over all ice surfaces it comes into contact with, as even the highest inferred temperature on Enceladus' surface $(145 \mathrm{~K})$ is still low 

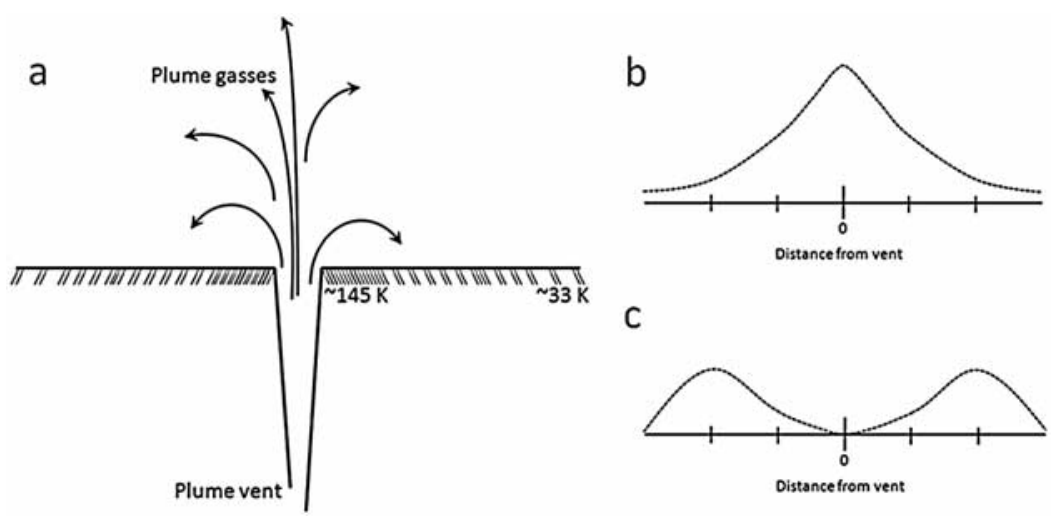

Figure 2. (a) A plume vent on Enceladus. The composition of the gasses condensed on the surface is related to the temperature of the surface, which is warmer closer to the vent. (b) Surface methanol concentration as a function of distance from the vent if methanol is deposited directly from the plume. (c) Surface methanol concentration as a function of distance from the vent if methane is deposited from the plume and photochemically converted to methanol.

enough for methanol to condense. Methanol concentrations should be highest immediately adjacent to the plume vents and decrease with increasing distance from the vent, as shown in Figure 2b. Scenario 2 is more complex: if the methanol is generated photochemically from methane then the amount of methane deposited becomes important. The coldest areas of Enceladus will trap more methane than warmer regions; the high temperatures of the tiger stripes themselves $(\sim 145 \mathrm{~K})$ will prevent methane from sticking. However, the amount of methane available for trapping in the ice will be greater closer to the vent. The balance between these two constraints will lead to a bimodal distribution, with the greatest concentration of surface methanol some distance from the plume vent (Figure 2c). Of course, some combination of these two processes may also occur, resulting in a hybrid distribution. Further study of the photochemical formation and degradation rates of methanol in water ice would enable the construction of a model of the processes described here. In combination with careful mapping of the strength and position of the methanol feature across Enceladus' surface, the model would constrain the origin of the methanol, and yield insights into the role of plume deposition and chemistry on Enceladus' surface.

[11] Acknowledgments. This work was performed at the Jet Propulsion Laboratory (JPL), California Institute of Technology, under a contract with the National Aeronautics and Space Administration (NASA). YLY was supported by NASA grant NNG06GF33G to the California Institute of Technology. Financial support through JPL's Research and Technology Development program is gratefully acknowledged. We thank Bonnie Buratti and James Bauer (JPL) for providing the Cassini VIMS data, and Arthur L. Lane (JPL) for useful discussions.

\section{References}

Bernstein, M. P., D. P. Cruikshank, and S. A. Sandford (2006), Nearinfrared spectra of laboratory $\mathrm{H}_{2} \mathrm{O}-\mathrm{CH}_{4}$ ice mixtures, Icarus, 181, 302, doi:10.1016/j.icarus.2005.10.021.

Brown, R. H., et al. (2006), Composition and physical properties of Enceladus' surface, Science, 311, 1425, doi:10.1126/science.1121031.

Brunetto, R., G. A. Baratta, M. Domingo, and G. Strazzulla (2005), Reflectance and transmittance spectra $(2.2-2.4 \mu \mathrm{m})$ of ion irradiated frozen methanol, Icarus, 175, 226, doi:10.1016/j.icarus.2004.10.011.

Cottin, H., M. H. Moore, and Y. Benilan (2003), Photodestruction of relevant interstellar molecules in ice mixtures, Astrophys. J., 590, 874, doi:10.1086/375149.

Emery, J. P., D. M. Burr, D. P. Cruikshank, R. H. Brown, and J. B. Dalton (2005), Near-infrared (0.8-4.0 $\mu \mathrm{m}$ spectroscopy of Mimas, Enceladus,
Tethys, and Rhea, Astron. Astrophys., 435, 353, doi:10.1051/00046361:20042482.

Grundy, W. M., B. Schmitt, and E. Quirico (2002), The temperature-dependent spectrum of methane ice I between 0.7 and $5 \mu \mathrm{m}$ and opportunities for near-infrared remote thermometry, Icarus, 155, 486, doi:10.1006/ icar.2001.6726.

Hodyss, R., P. V. Johnson, J. V. Stern, J. D. Goguen, and I. Kanik (2009) Photochemistry of methane-water ices, Icarus, 200, 338, doi:10.1016/ j.icarus.2008.10.024.

Hudgins, D. M., S. A. Sandford, L. J. Allamandola, and A. G. G. M. Tielens (1993), Mid- and far-infrared spectroscopy of ices-Optical constants and integrated absorbances, Astrophys. J. Suppl. Ser., 86, 713, doi:10.1086/191796.

Loeffler, M. J., and R. A. Baragiola (2009), Is the $3.5 \mu \mathrm{m}$ infrared feature on Enceladus due to hydrogen peroxide?, Astrophys. J., 694, L92, doi:10.1088/0004-637X/694/1/L92.

Loeffler, M. J., U. Raut, and R. A. Baragiola (2006), Enceladus: A source of nitrogen and an explanation for the water vapor plume observed by Cassini, Astrophys. J., 649, L133, doi:10.1086/508459.

Madey, T. E., R. E. Johnson, and T. M. Orlando (2002), Far-out surface science: Radiation-induced surface processes in the solar system, Surf. Sci., 500, 838, doi:10.1016/S0039-6028(01)01556-4.

Matson, D. L., J. C. Castillo, J. Lunine, and T. V. Johnson (2007), Enceladus' plume: Compositional evidence for a hot interior, Icarus, 187, 569, doi:10.1016/j.icarus.2006.10.016.

Newman, S. F., B. J. Buratti, R. Jaumann, J. M. Bauer, and T. W. Momary (2007), Hydrogen peroxide on Enceladus, Astrophys. J., 670, L143, doi:10.1086/524403.

Palumbo, M. E., A. C. Castorina, and G. Strazzulla (1999), Ion irradiation effects on frozen methanol $\left(\mathrm{CH}_{3} \mathrm{OH}\right)$, Astron. Astrophys., 342, 551.

Parkinson, C. D., et al. (2007), Enceladus: Cassini observations and implications for the search for life, Astron. Astrophys., 463, 353, doi:10.1051/0004-6361:20065773.

Sandford, S. A., and L. J. Allamandola (1993), Condensation and vaporization studies of $\mathrm{CH}_{3} \mathrm{OH}$ and $\mathrm{NH}_{3}$ ices: Major implications for astrochemistry, Astrophys. J., 417, 815, doi:10.1086/173362.

Spencer, J. R., et al. (2006), Cassini encounters Enceladus: Background and the discovery of a south polar hot spot, Science, 311, 1401, doi:10.1126/ science.1121661.

Strazzulla, G., G. Leto, F. Spinella, and O. Gomis (2005), Production of oxidants by ion irradiation of water/carbon dioxide frozen mixtures, $A s$ trobiology, 5, 612, doi:10.1089/ast.2005.5.612.

Waite, J. (2008), Enceladus plume composition, Eos Trans. AGU, 89(53), Fall Meet. Suppl., Abstract P14A01.

Waite, J. H., et al. (2006), Cassini ion and neutral mass spectrometer: Enceladus plume composition and structure, Science, 311, 1419, doi:10.1126/science. 1121290 .

J. D. Goguen, R. Hodyss, P. V. Johnson, I. Kanik, and J. V. Stern, Jet Propulsion Laboratory, California Institute of Technology, Pasadena, CA 91109, USA. (robert.p.hodyss@jpl.nasa.gov)

C. D. Parkinson, Department of Atmospheric, Oceanic, and Space Sciences, University of Michigan, Ann Arbor, MI 48109, USA.

Y. L. Yung, Division of Geological and Planetary Sciences, California Institute of Technology, Pasadena, CA 91125, USA. 